NASA Technical Memorandum 103928

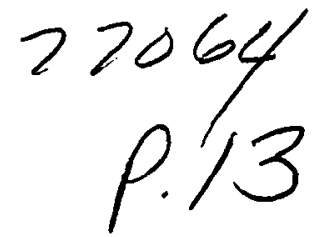

\title{
Laser-Spectroscopic Measurement Techniques for Hypersonic, Turbulent Wind Tunnel Flows
}

\section{Robert L. McKenzie and Douglas G. Fletcher}

March 1992

(NASA-TM-103928) LASER-SPECTROSCOPIC MEASUREMENT TECHNIQUES FOR HYPERSONIC, TURBULENT WIND TUNNEL FLOWS (NASA) $13 \mathrm{p}$
N92-19596

Unclas G3/35 0077064 


\section{Laser-Spectroscopic Measurement Techniques for Hypersonic, Turbulent Wind Tunnel Flows}

Robert L. McKenzie and Douglas G. Fletcher, Ames Research Center, Moffett Field, California

March 1992

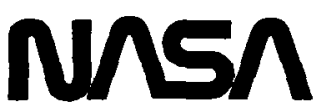

National Aeronautics and

Space Administration 


\title{
LASER-SPECTROSCOPIC MEASUREMENT TECHNIQUES FOR HYPERSONIC, TURBULENT WIND TUNNEL FLOWS
}

\author{
ROBERT L. MCKENZIE AND DOUGLAS G. FLETCHER \\ NASA Ames Research Center \\ Moffett Field, California, USA
}

\section{Introduction}

Until recently, aerodynamic measurements obtained away from a surface have been limited either to the use of intrusive probes to determine pressure, mass flux, and total temperature or to visualization methods for indications of the flow geometry. New laser-spectroscopic methods are now emerging which offer compelling opportunities to obtain simultaneous measurements of density, temperature, and their fluctuations, with unprecedented spatial and temporal resolution [1]. As a result, measurement techniques are now feasible which can be used in supersonic and hypersonic flows to characterize the effects of compressibility on turbulent behavior and to obtain quantitative descriptions of complex, three-dimensional flow fields.

Most of the laser-spectroscopic techniques incorporate laser-induced fluorescence (LIF) or Raman spectroscopy. The primary objective of this report is to describe the spectroscopic nature, present status, and capabilities of some LIF and Raman techniques which have been developed at NASA Ames Research Center. Particular emphasis has been centered on applications in hypersonic wind tunnels where measurements with more conventional probes are often inadequate or impractical. The requirements for laser-spectroscopic measurements in hypersonic flows are dictated by their intended aerodynamic rescarch applications. By design, hypersonic flow fields over test articles are generally free of large stagnation regions. Consequently, in moderately heated facilities, they can have densities lower than 0.01 amagat and temperatures ranging from $50 \mathrm{~K}$ to $300 \mathrm{~K}$. To provide sufficient sensitivity at the low temperatures, the spectroscopy must be based on individual rotational transitions in the interacting species. In addition, most applications are generally limited to chemically stable species which have been seeded into the flow or which occur naturally in air.

With these requirements in mind, two principal laser-spectroscopic approaches have been developed at NASA Ames Research Center for the measurement of temperature, density, and their fluctuations owing to turbulence in high-speed flows. Early work was centered on the use of near-ultraviolet lasers to induce fluorescence from trace concentrations of nitric oxide (NO) added to flows of nitrogen. More recently, advances in laser technology have allowed similar measurements to be made in unseeded air flows using vacuum-ultraviolet lasers to excite molecular oxygen. 


\section{Laser-Induced Fluorescence in Flows Containing Nitric Oxide}

Prior to the availability of high-energy excimer lasers, a spectroscopic approach was developed which relied on the existing Nd:YAG-pumped, tunable dye laser technology. It was based a dualfrequency LIF technique in which two frequency-doubled dye lasers were each tuned to a nearultraviolet, ro-vibronic transition in the Gamma Band spectrum of nitric oxide [2]. Pulse energies of $1 \mu \mathrm{J}$ could be achieved over the entire electronic band near $225 \mathrm{~nm}$. The two independent, coincident LIF signals allowed density and temperanure to be determined simultaneously. NO was added in concentrations of less than $100 \mathrm{ppm}$ to a wind tunnel reservoir containing pure nitrogen. Because the NO fluorescence is quenched efficiently by oxygen and very little by nitrogen, the technique is most applicable in pure nitrogen flows.

The capabilities of the method were demonstrated in the turbulent boundary layer on the wall of a Mach-2 channel flow. Single-pulse measurements were obtained with a spatial resolution of less than $1 \mathrm{~mm}$ and uncertainties of less than $2 \%$. A small blow-down wind tunnel was used which was designed to handle gas mixtures containing NO. Since NO is toxic in concentrations greater than 25 ppm, a test facility suitable for NO-LIF must incorporate all of the special feanures necessary to safely use toxic mixtures. Despite this inconvenience for general applications, the NO-LIF technique demonstrated the unique capabilities of laser-spectroscopic methods for aerodynamic measurements. Its success encouraged investigations of similar techniques which could be applied to unseeded air flows.

\section{Raman Scattering and Laser-Induced Fluorescence in Air Flows}

\subsection{BACKGROUND}

The development of high energy, vacuum-ultraviolet, ArF excimer lasers made feasible the use of LIF from oxygen molecules in air. Exploration of the concept was initiated in 1981, before such lasers were available commercially, with a study solicited by NASA-Ames and performed by Massey and Lemon [3]. They were able to construct a tunable, ArF laser and demonstrate its use to obtain $\mathrm{O}_{2}$ LIF signals in room air. Their measurements indicated that the LIF was sufficiently intense and sensitive to temperature to be a viable measurement technique in unseeded air flows.

The results of Masscy and Lemon and the subsequent commercial availability of high-energy ArF excimer lasers encouraged further development of the concept. However, unlike the twolaser system used for NO-LIF, the cost and complexity of excimer lasers made the use of two lasers unattractive. Altematively, Gross and McKenzie [4] proposed that both the fluorescence emission and the Raman scattering from each pulse of one laser be used to provide the two independent signals required for simultaneous temperature and density measurements. Since then, Laufer et al. have characterized the spectroscopy of $0_{2}$-LIF [5] and demonstrated its use to measure temperatures down to $130 \mathrm{~K}$ in a cell at known pressures [6]. The development was continued by Fletcher who incorporated the Raman signal to obtain simultaneous measurements of temperatures and densities, without knowledge of any other thermodynamic parameters [7]. Most recently, he has demonstrated the use of the $0_{2}$-LIF/Raman technique to measure temperature, density, and their fluctuations in the turbulent boundary layer of the same Mach-2 channel flow which was studied previously using NO-LIF [8]. 


\subsection{2-LIF/RAMAN SPECTROSCOPY}

The $\mathrm{O}_{2}$ LIF process is characterized by the energy-level diagram shown in Fig. 1. The uning range of the ArF laser is centered at $193.3 \mathrm{~nm}$ with a bandwidth of approximately $0.5 \mathrm{~nm}$. Within that band, absorbing transitions may be accessed in the $0_{2} B^{3} \Sigma_{u}^{-} \leftarrow X^{3} \Sigma_{B}^{-}$Schumann-Runge band from $v^{\prime \prime}=0$ to $v^{\prime}=4,5$. Hot-band vibrational transitions are also accessible, including $v^{\prime \prime}=1$ to $v^{\prime}=7,8$; and $v^{\prime \prime}=2$ to $v^{\prime}=10,11,12$. In each vibrational band, the rotational transition pairs, $P\left(K^{\prime \prime}\right)$ and $R\left(K^{n}+2\right)$, are at nearly the same frequency. The pairs associated with $K^{n}=15,17$, and 19 from $v^{\prime}, v^{\prime \prime}=4,0$ have been the principle absorbing transitions used for LIF at room temperature and below. Below room temperature, the population of levels with $v^{n}>0$ is extremely small. However, the Frank-Condon factor for $v^{\prime}, v^{\prime \prime}=7,1$ transitions is 200 times greater than for $v^{\prime}, v^{\prime \prime}=4,0$ so that only a small population of the $v^{\prime \prime}=1$ level is necessary to produce observable hot-band absorption. The effect starts to become significant at temperatures slightly above $300 \mathrm{~K}$.

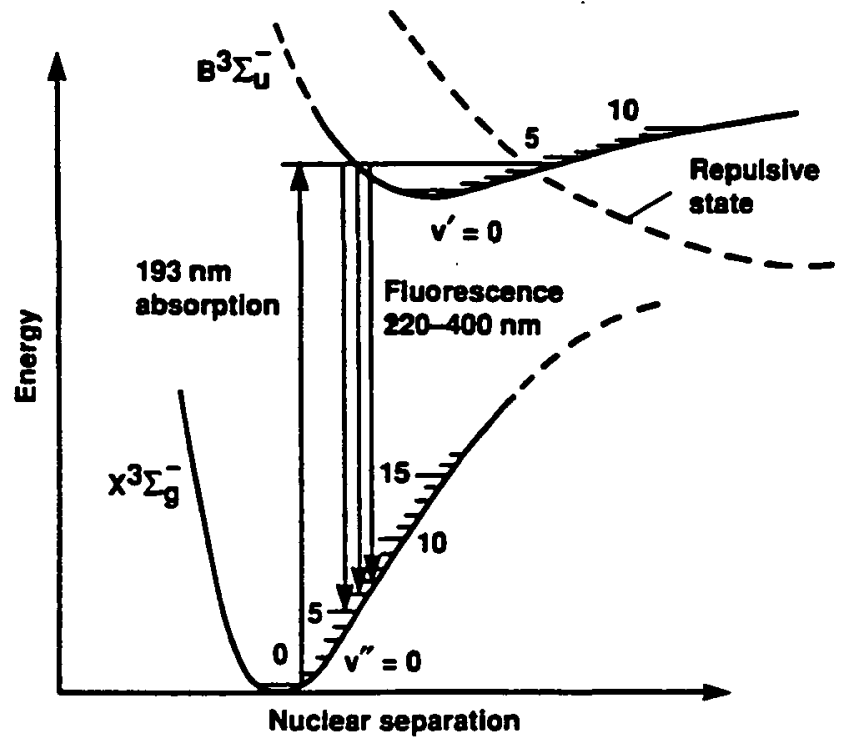

Figure 1. Energy-level diagram for $\mathrm{O}_{2}$ laser-induced fluorescence by an ArF laser.

The $\mathrm{B}^{3} \Sigma_{\mathrm{u}}^{-}$upper statc of $\mathrm{O}_{2}$ is crossed near the $\mathrm{v}^{\prime}=4$ level by an array of repulsive states which are represented by a single curve in Fig. 1. Consequently, most molecules which are elevated to $v^{\prime} \geq 4$ by the LIF process predissociate within a few picoseconds. The molecules that do not predissociate must have a radiative lifetime which is equally short. Consequently, it is much less than the time between molecular collisions at most wind tunnel test conditions. Collisional quenching effects on the LIF signals may then be neglected, thereby greatly simplifying the signal analysis required to determine temperature and density.

For fluorescence originating from $v^{\prime}=4$, the Franck-Condon factors favor spontaneous transitions back to all $X^{3} \Sigma_{g}^{-}$vibrational levels above $v^{n}=4$. The resulting fluorescence spectrum extends from $220 \mathrm{~nm}$ to greater than $400 \mathrm{~nm}$. Although the total fluorescence signal is maximized if the entire band is observed, spectral interference from other laser-induced processes can limit the practical range of observation. In any case, at least half of the fluorescence energy is from wavelengths below $300 \mathrm{~nm}$, which includes $0_{2}$ vibrational emission bands up to $v^{\prime}, v^{\prime \prime}=4,13$. 
The LIF emission provides a signal which is sensitive to both temperaure and density. The Raman signal can be made sensitive only to density. Taken together, the two contributions to the laser-induced spectrum allow temperature and density to be determined from each laser pulse.

An experimental spectrum of laser-induced radiation from air at room temperature is shown in Fig. 2. It was obtained using an optical multi-channel analyzer (OMA) at the exit plane of a small spectrometer with a $0.5-\mathrm{mm}$ entrance slit. Because the image of the laser beam lies with its axis orthogonal to the slit, the slit width also determines the length of the observed sample volume. In this example, the spectral width of each feature is dominated by the width of the slit image. In addition, the grating has been positioned so that the contribution from Rayleigh scattering below $196 \mathrm{~nm}$ does not illuminate the OMA photo-diode array. The $\Delta v$ notation refers to the overall change in vibrational quantum number resulting from the entire Raman or LIF process. The features denoted by $\Delta v=1,2$ are the Raman fundamental and first overtone transitions of $\mathrm{O}_{2}$. The Raman $\Delta v=1$ transition from $\mathrm{N}_{2}$ falls between them. The strengths of the $0_{2}$ Raman scattering are resonantly enhanced at $193 \mathrm{~nm}$, which significantly improves the viability of Raman measurements in air at low densities. At room temperature and below, the fluorescence underlying the $0_{2} \Delta v=1$ Raman transition is negligible because the $v^{\prime}, v^{\prime \prime}=4,1$ Frank-Condon factor is so small. Hence, the $\Delta v=1$ transitions of $\mathrm{O}_{2}$ and $\mathrm{N}_{2}$ may be assumed to result only from Q-branch Raman transitions for which all rotational components are at nearly the same frequency. The resulting, spectrally integrated signal is independent of temperature and is linearly proportional to the species density. The sum of $\mathrm{O}_{2}$ and $\mathrm{N}_{2}$ Raman contributions provide a maximum signal for determining the gas density. The $Q_{2} \Delta v=2$ feature is not included in the sum to avoid the small influence of underlying fluorescence.

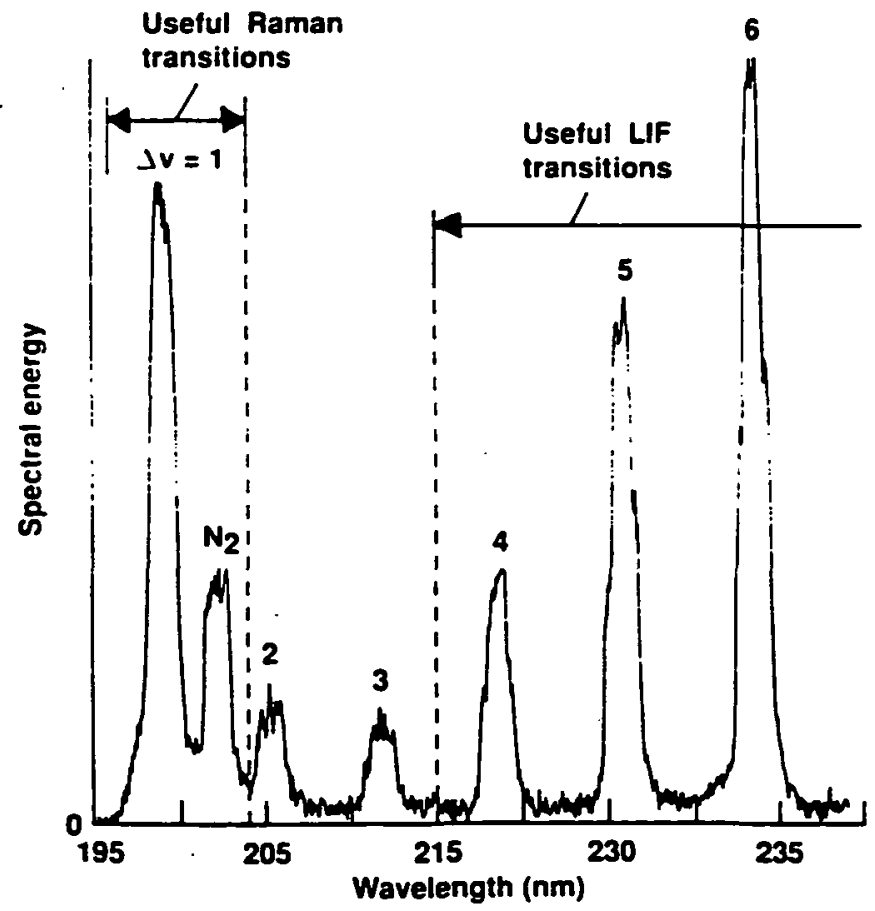

Figure 2. Emission spectrum from room air induced by an ArF laser with low fluence. 
The LIF transitions at the wavelengths captured by the OMA diode array also appear in Fig. 2. The entire useful spectrum extends to $\Delta v=13$ at $300 \mathrm{~nm}$. The $\Delta v=3$ transition is avoided because it contributes only a small fraction to the total fluorescence energy and includes a weak contribution from the second overtone of the resonance-enhanced $\mathrm{O}_{2}$ Raman scattering.

Because the laser energy typically fluctuates $\pm 5 \%$ from pulse to pulse, the Raman and LIF spectral energies for each pulse must be normalized by the corresponding laser energy. However, only the laser pulse energy can be measured accurately, but not its fluence (energy per unit area). Consequently, the observed radiative processes must be dominated by interactions for which the signal energies vary linearly with laser energy and are independent of the distribution of fluence within the beam cross section. The dominance of linear LIF processes depends both on the magnitude of laser fluence and on its wavelength. For example, Fig. 3 shows that as the laser fluence exceeds about $1.5 \mathrm{~J} / \mathrm{cm}^{2}$, both the Raman and LIF energy behave quadratically, depending on the transition excited. This behavior is the consequence of underlying multi-photon processes with excitation rates that increase non-linearly with laser fluence and become dominant at high values.

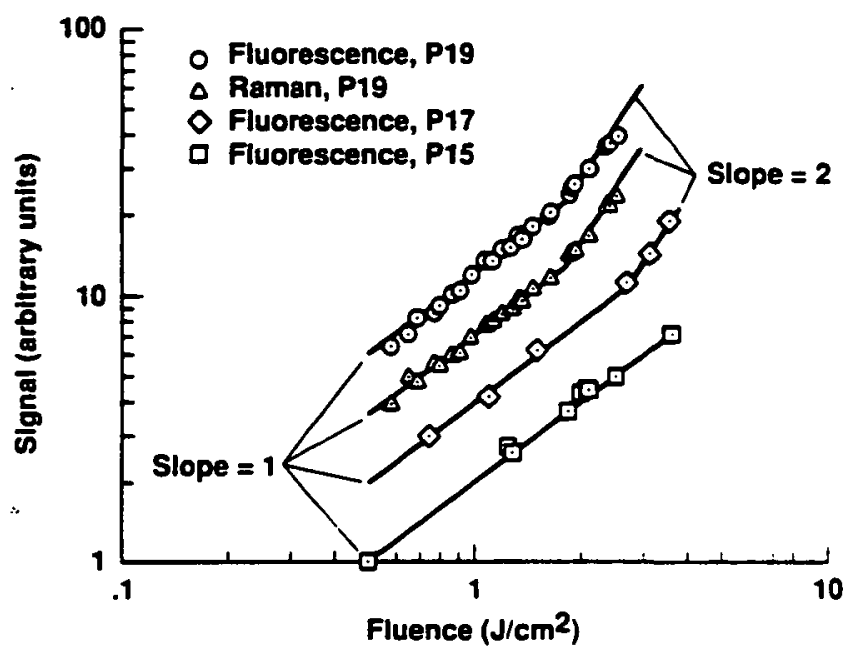

Figure 3. Dependence of total fluorescence and Raman signals on the spatially-averaged fluence of a narrowband ArF laser. (Note: The signal magnitudes for each case have been arbitrarily separated for clarity.)

The dependence of the non-linear processes on laser wavelength is demonstrated more explicitly in Fig. 4. It shows an excitation spectrum in which the total LIF energy is monitored while the laser is tuned through the absorbing $\mathrm{O}_{2}$ transitions. The laser has been focused to achieve a high average fluence. The non-linear resonances, denoted by asterisks, do not appear when an unfocused beam of the same pulse energy is used. Most of them have been identified as resonances associated with the photoion, $0_{2}^{+}$. The resonant wavelengths of the non-linear processes influence the choice of $\mathrm{O}_{2}$ transitions used to obtain LIF measurements. For example, the P19 and R21 transitions are both strongly affected. Interference underlying the Raman transitions also occurs at this laser wavelength, as shown by Fig. 3. The P17 transition can be affected similarly if the laser is not carefully tumed or sufficiently narrowband. The nearby R19 transition produces weaker LIF signals than those from P17 but lies farther from the non-linear resonance. Finally, the P15 transition is apparently free of nearby non-linear resonances for the range of fluence investigated. 


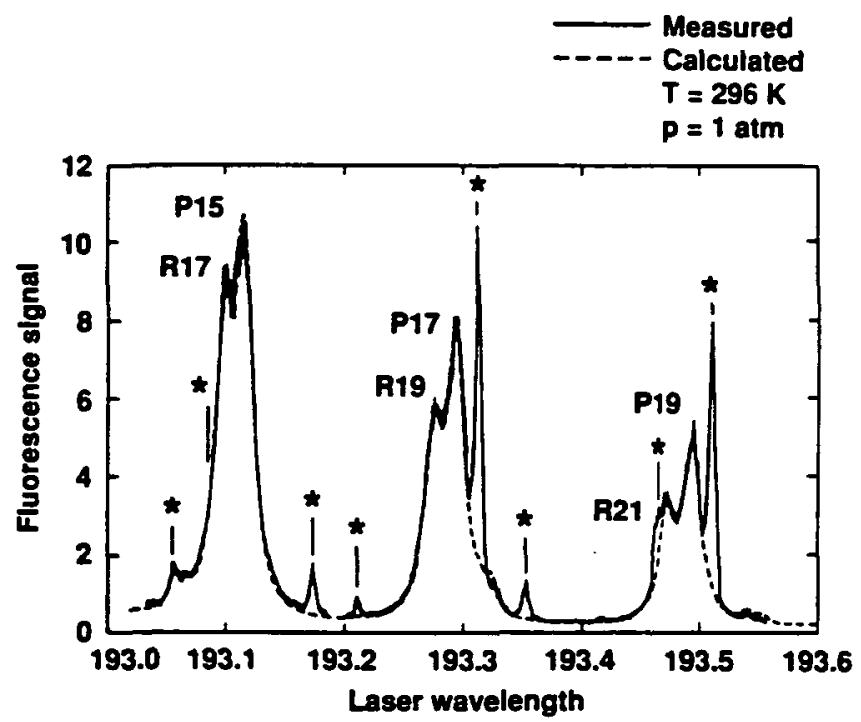

Figure 4. Oxygen excitation spectrum stimulated by a focused laser beam with an average fluence of about $1.5 \mathrm{~J} / \mathrm{cm}^{2}$. Multi-photon transitions are denoted by asterisks.

\subsection{APPLICATION TO A MACH-2 TURBULENT BOUNDARY LAYER}

Work is now in progress to demonstrate the use of the $\mathrm{O}_{2}$-LIF/Raman technique for simultaneous measurements of temperatures, densities, and their fluctuations owing to supersonic turbulence [8]. The characteristics of the test flow have been described previousiy [2-3]. It includes a 12.1-mm thick, fully turbulent boundary layer that is adjoined by a relatively undisturbed flow of the same thickness.

In Fig. 5, the average temperature and density profiles obtained from $\mathrm{O}_{2}$-LIF/Raman data are compared with distributions implied from pressure measurements using a pitot probe and a static pressure port in the wall below the sample volume. Spatial resolution of the spectroscopic measurements was about $1 \mathrm{~mm}$. Agreement of comparable data is within $\pm 3 \%$. Since the flow is in thermochemical equilibrium and both state variables have been measured simultaneously, the $\mathrm{O}_{2}$-LIF/Raman data may also be used in conjunction with the cquation of state to determine the local pressure. The pressure distribution is nearly constant, as expected for a flat-wall boundary layer. These results are identical to those obtained previously using NO-LIF.

Figure 6 shows a comparison of the root-mean-square ( $\mathrm{ms}$ ) fluctuation amplitudes of temperature and density obtained from both spectroscopic methods and from hot-wire data. Again, the NO-LIF and $\mathrm{O}_{2}-\mathrm{LIF} / \mathrm{Raman}$ results are in agreement. They both show a region in the boundary layer near $Y / \delta=0.6$ where the density fluctuations are exceptionally large. A histogram of the NO-LIF fluctuations displays a bimodal character which suggests that a weak wave was oscillating across the sample volume. Such density fluctuations also imply the presence of pressure fluctuations in that region. On the other hand, an analysis of the hot-wire signals to obtain density fluctuations requires the assumption that pressure fluctuations are negligible. Clearly, that is not the case in this flow and the effects of pressure fluctuations would not have been observed without the use of the LIF instrumentation. These comparisons demonstrate that 
the $\mathrm{O}_{2}$-LIF/Raman technique can provide accurate measurements in air flows at the low temperatures and densities which are prevalent in high-speed wind tunnels.
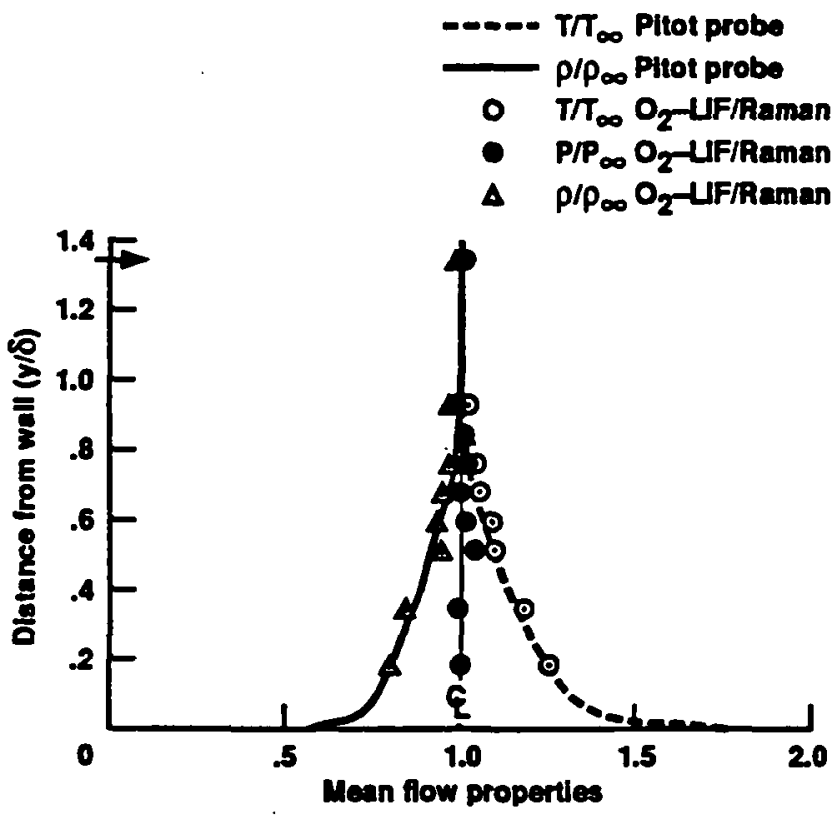

Figure 5. Time-averaged temperature, density, and pressure distributions in a turbulent boundary layer with a centerline Mach number $\mathrm{M}_{\infty}=2.06$, stream temperature $\mathrm{T}_{\infty}=158 \mathrm{~K}$, stream pressure $P_{\infty}=0.35 \mathrm{~atm}$, and thickness $\delta=12.1 \mathrm{~mm}$. Local values are normalized by their respective free stream values.

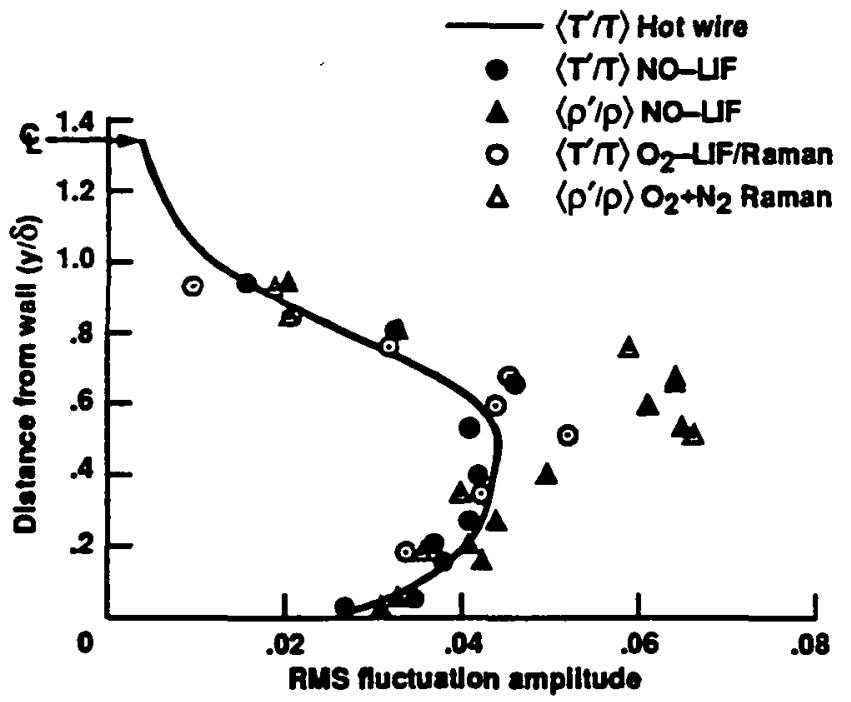

Figure 6. Comparisons of temperature and density ms-fluctuation amplitudes obtained with LIF techniques or implied by hot-wire anemometer data. Flow conditions are the same as in Fig. 5. For each variable, the fluctuation amplitude is normalized by the local time-averaged value. 


\section{Applications to Large-Scale Hypersonic Flows}

A compelling application of the $\mathrm{O}_{2}$-LIF/Raman technique is to flows in the NASA-Ames $3.5 \mathrm{ft}$. Hypersonic Wind Tunnel. Its test region is a open free-jet at Mach numbers of 5, 7,and 10, depending on the nozzle. The test environment serves as a relevant example for evaluations of the capabilities of $\mathrm{O}_{2}$-LIF/Raman techniques.

LIF measurements have been demonstrated in which uncertainties greater than $1 \%$ may be characterized solely by the unavoidable effects of photon-statistical noise in the radiative signals [6]. In that case, the uncertainty or ms-noise/signal ratio for pulsed signals is simply equal to $\left[n_{e}\right]^{-1 / 2}$, where $n_{e}$ is the average number of photo-electrons detected per pulse. This feature allows the corresponding uncertainties in temperature and density to be predicted in terms of the laser pulse energy and the efficiencies of the detector and collection optics. It may also be used to extend predictions based on the design parameters chosen used here to other values of pulse energy, f-number, etc.

For a given temperature, the weakest LIF signals are always obtained at the lowest densities. The density, $p=0.02$ amagat, is representative of the free-stream density at Mach 7. To evaluate the capabilities of $\mathrm{O}_{2}$-LIF/Raman measurements, the corresponding uncertainties in temperature measurement may be calculated using the most favorable set of optical parameters believed achievable and assuming that the optical system accepts fluorescence emission from all upper states with $\Delta v \leq 11$. In practice, more restrictive filtering of the emission spectrum may be required, depending on the experimental arrangement.

Among the significant parameters related to the performance of the collection optics system are its effective f-number and the transmission and reflection losses of its optical components. A system design based on $\mathrm{f} / 2$ optics, for which only $1.5 \%$ of the total radiative emission is collected, will still require a large device to match the focal lengths necessary in a large-scale facility. For example, in the Ames hypersonic facility, the optics can not be much closer to the flow centerline than $1 \mathrm{~m}$, thereby requiring an $\mathrm{f} / 2$ system to have an aperture diameter of $50 \mathrm{~cm}$. If a Cassegrain telescope is used, its two mirrors typically provide a combined reflectivity of $75 \%$ at $200 \mathrm{~nm}$. In addition, the mirrors must be protected from heat and dust from the free-jet flow by several window panes of fused silica. Their combined transmission, including absorption and surface reflection losses, will be near $60 \%$. Finally, isolation of the Raman spectra requires a monochromator with at least a grating, even if all other mirrors are eliminated. Grating efficiencies near $200 \mathrm{~nm}$ are typically less than $50 \%$. Finally, losses at the monochromator entrance slit owing to imperfect imaging can exceed $10 \%$. Thus, an overall transmission of no better than $20 \%$ should be expected. This value, combined with the collection efficiency of $f / 2$ optics and a representative detector quantum efficiency of $12 \%$, leads to an anticipated efficiency for conversion of emitted photons to detected photoelectrons of only $1 / 3000$.

The laser pulse used for the calculations has been limited to $15 \mathrm{~mJ}$, to stay within the range of fluence for linear behavior with a $1-\mathrm{mm}$ spatial resolution at all laser wavelengths. However, linear behavior has been demonstrated for excitation of the P15 transition with pulse energies to $30 \mathrm{~mJ}$, as Fig. 3 indicates.

The effect of the Raman signal uncertainty is also included in the temperature uncertainty calculation since the LIF signals are proportional to density. For the parameters given, the density measurements will contain a 5\% ms-uncertainty owing to photon-statistical noise. However, density fluctuations owing to turbulence at hypersonic speeds are expected to be larger.

The variations in temperature measurement uncertainty are shown in Fig. 7 for narrowband laser wavelengths which are resonant with the absorbing transitions indicated. At the lowest 
temperatures, the P15 transition is superior and can be further enhanced in practice by using higher pulse energies. However, its useful range of temperatures is limited and superseded by the $R 19$ transition above $140 \mathrm{~K}$. The P17 transition is not attractive at low temperatures because it is most favorable in only a limited temperature range and susceptible to non-linear interference. Above $300 \mathrm{~K}$, the sensitivity of any $v^{\prime}, v^{\prime \prime}=4,0$ transition must be compared with that available from excitation of the hot-band $v^{\prime}, v^{\prime \prime}=7,1$ transitions [6].

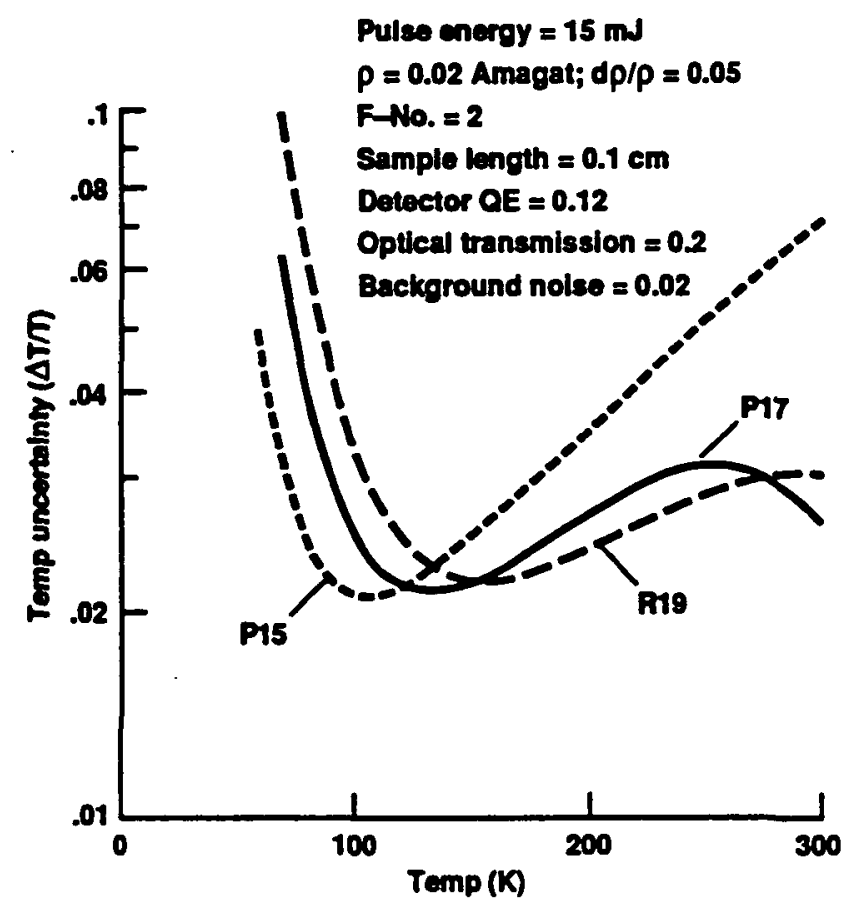

Figure 7. Predictions of temperature measurement uncertainties owing to photon-statistical noise for constant density and laser pulse energy.

\section{Conclusions}

These results demonstrate that laser-spectroscopic techniques can be used to obtain measurements of temperature, density, and their turbulent fluctuations and they show the feasibility of using a carefully tuned, narrowband ArF excimer laser and $\mathrm{O}_{2}$-LIF/Raman spectroscopy to measure temperatures and densities at hypersonic wind tunnel conditions with uncertainties of less that 5\%. 


\section{References}

1. McKenzie, R. L. (1991) "Progress in Laser-Spectroscopic Techniques for Aerodynamic Measurements-An Overview," AIAA Paper 91-0059.

2. Gross, K. P., McKenzie, R. L., and Logan, P. (1987) "Measurements of Temperature, Density, Pressure, and Their Fluctuations in Supersonic Turbulence Using Laser-Induced Fluorescence," Exp. in Fluids, 5 pp. 372-380.

3. Massey, G. A. and Lemon, C. J. (1984) "Feasibility of Measuring Temperature and Density Fluctuations in Air Using Laser-Induced $\mathrm{O}_{2}$ Fluorescence," IEEE Joum. Quantum Elect., QE-20, pp. 454-457.

4. Gross, K. P. and McKenzie, R. L. (1985) "Optical Diagnostics of Unseeded Turbulent Flows Using Ultraviolet Laser-Induced Processes in Air," Unpublished Research Proposal.

5. Laufer, G., McKenzie, R. L., and Huo, W. M. (1988) "Radiative Processes in Air Excited by an ArF Laser," Optics Lett, 13, pp. 99-101.

6. Laufer, G., McKenzie, R. L., and Fletcher, D. G. (1990) "Method for Measuring Temperatures and Densities in Hypersonic Wind Tunnel Air Flows Using Laser-Induced $\mathrm{O}_{2}$ Fluorescence," Applied Optics, 29, pp. 4873-4883.

7. Fletcher, D. G. and McKenzie, R. L. (1991) "Simultaneous Measurements of Temperature and Density in Air Flows Using UV Laser Spectroscopy," AIAA Paper 91-0458.

8. Fetcher, D. G. (1992) "Measurements of Density, Temperature, and Their Fluctuations in Turbulent, Supersonic Flow Using UV Laser Spectroscopy." Unpublished presentation at the AIAA 30th Aerospace Sciences Meeting, Reno, NV. 
Public reponting burdon for this collection of information bestimated to average 1 hour per response, Inctuding the time for roviewing inatructions, eearching existing dala sources, gathering and malntaining the dara needed, and completting and revlewing the collection of information. Send comments regarding thls burden estimate or any other aspect of this Davks Hghway, Sulte 1204, Arlington. VA 22202-4302, and to the Otflce of Managemem and Budget, Paperwork Reduction Project (0704-0188), Washington, DC 20503.
1. AGENCY USE ONLY (Leave blank)
2. REPORT DATE
March 1992
3. REPORT TYPE AND DATES COVERED
Technical Memorandum

4. TITLE AND SUBTITLE

Laser-Spectroscopic Measurement Techniques for Hypersonic, Turbulent

Wind Tunnel Flows

6. AUTHOR(S)

$505-59-40$

Roben L. McKenzie and Douglas G. Fletcher

7. PERForming ORganization maME(S) AND ADdRESS(ES)

8. PERFORMING ORGANIZATION REPORT NUMBER

Ames Research Center

Moffett Field, CA 94035-1000

A-92076

9. SPONSORING/MONITORING AGENCY NAME(S) AND ADDRESS(ES)

10. SPONSORING/MONITORING AGENCY REPORT NUMBER

National Aeronautics and Space Administration

Washington, DC 20546-0001

NASA TM-103928

11. SUPPLEMENTARY NOTES

Point of Contact: Robert L. McKenzie, Ames Research Center, MS 229-1, Moffett Field, CA 94035-1000; (415) $604-6158$ or FTS $464-6158$

12a. DISTRIBUTION/AVAILABILITY STATEMENT

12b. DISTRIBUTION CODE

Unclassified - Unlimited

Subject Category 35

13. ABSTRACT (Maximum 200 words)

A review is given of the nature, present status, and capabilities of two laser-spectroscopic methods for the simultaneous measurement of temperature, density, and their fluctuations owing to turbulence in high speed wind tunnel flows. One method is based on the two-frequency excitation of nitric oxide seeded into a nitrogen flow, using tunable dye lasers. The second, more recent method relies on the excitation of oxygen in air flows using a tunable, ArF excimer laser. Signals are obtained from both the laser-induced fluorescence and from Raman scattering of the same laser pulse. Measurements are demonstrated in the turbulent boundary layer of a Mach-2 channel flow.

14. SUBJECT TERMS

Hypersonic flow measurements, Turbulent flow measurements, Laser-induced fluorescence, Flow diagnostics, Oxygen fluorescence, Temperature measurements, Density measurements

17. SECURITY CLASSIFICATION OF REPORT

18. SECURITY CLASSIFICATION OF THIS PAGE

Unclassified

Unclassified
15. NUMBER OF PAGES

14

16. PRICE CODE

$\mathrm{AO2}$ 